\title{
Adipose-Derived Stem Cells Stimulate Reendothelialization in Stented Rat Abdominal Aorta
}

Tomohiko Sato, MD, PhD; Masao Takahashi, MD, PhD; Daishi Fujita, MD, PhD; Shigeyoshi Oba, MD, PhD; Hiroaki Nishimatsu, MD, PhD; Tetsuo Nagano, PhD; Etsu Suzuki, MD, PhD

\begin{abstract}
Background: Although drug-eluting stents (DES) have been widely used for the treatment of coronary artery disease, they potentially increase the risk of late thrombosis. It is, therefore, desirable to establish a strategy to stimulate reendothelialization. Endothelial injury models have been widely used to analyze the mechanisms of coronary restenosis. However, animal models deployed with coronary stents in the blood vessels are necessary to accurately analyze the mechanisms of coronary restenosis and late thrombosis because persistent inflammation occurs around the coronary stents.
\end{abstract}

\begin{abstract}
Methods and Results: Coronary stents were implanted into rat abdominal aorta and adipose tissue-derived stem cells (ASC) were administered from the adventitial side. Reendothelialization was then visualized by Evans blue staining, and neointimal formation was analyzed histologically. ASC significantly stimulated reendothelialization and inhibited neointimal formation in bare metal stents (BMS)-implanted aorta. In addition, ASC promoted reendothelialization in DES-implanted aorta; however, the effects were weaker than in BMS-implanted aorta. Among the cytokines that ASC produce, adrenomedullin (AM) significantly stimulated reendothelialization and inhibited neointimal formation in BMS-implanted aorta, when an adenovirus expressing AM was administered from the adventitial side.

Conclusions: These results suggest that ASC produce several cytokines that stimulate reendothelialization and inhibit neointimal formation in stent-deployed vessels, and that AM could mediate these effects. (Circ J 2014; 78: 1762-1769)
\end{abstract}

Key Words: Adipose tissue-derived stem cells; Adrenomedullin; Coronary stent; Neointimal formation; Reendothelialization

D rug-eluting stents (DES) have been widely used for the treatment of coronary artery disease and they are definitely useful for the inhibition of restenosis compared with bare metal stents (BMS) over the short- to mid-term period. However, the use of DES does not always improve the outcome of patients over a long-term period. This is probably because DES potentially increase the risk of late thrombosis. ${ }^{1,2}$ This complication might be due to the suppression of vascular endothelial cell (VEC) proliferation as well as vascular smooth muscle cell (VSMC) proliferation by antiproliferative drugs, resulting in the delayed reendothelialization of stented vessels and thrombus formation. Because VECs produce several factors such as prostacyclines and C-type natriuretic peptide that inhibit the proliferation of VSMCs, ${ }^{3,4}$ stimulation of reendothelialization might be a promising strategy to inhibit restenosis and late thrombosis. For this purpose, endothelial progenitor cell (EPC)capturing stents, which are coated with anti-CD34 antibody to capture EPCs on the surface of stents, have been clinically used.

\section{Editorial p1582}

Adipose tissue-derived stem cells (ASC) are mesenchymal stem cells that exist in subcutaneous adipose tissue in large quantities. ${ }^{5}$ ASC were clinically used to treat patients with critical limb ischemia, which turned out to be effective. ${ }^{6} \mathrm{We}$ recently demonstrated that ASC inhibited neointimal formation in a wire injury model of the rat femoral artery. ${ }^{7}$ ASC administration from the adventitial side also suppressed neointimal formation and stimulated reendothelialization significantly, suggesting that ASC inhibited neointimal formation in a paracrine manner. We have reported that ASC produce angiopoietin-1 (Ang-1) and that Ang-1 is implicated in ASC-induced stimulation of reendothelialization in the wire injury model. ${ }^{8}$ Furthermore, we have shown that ASC restore erectile function in diabetic rats and

Received January 5, 2014; revised manuscript received February 21, 2014; accepted March 13, 2014; released online April 24 , 2014 Time for primary review: 26 days

Department of Internal Medicine, Faculty of Medicine (T.S., M.T., D.F., S.O.), Department of Urology, Faculty of Medicine (H.N.), Graduate School of Pharmaceutical Sciences (T.N.), University of Tokyo, Tokyo; St. Marianna University School of Medicine, Kawasaki (E.S.), Japan

Mailing address: Etsu Suzuki, MD, PhD, Institute of Medical Science, St. Marianna University School of Medicine, 2-16-1

Sugao, Miyamae-ku, Kawasaki 216-8512, Japan. E-mail: esuzuki-tky@umin.ac.jp

ISSN-1346-9843 doi:10.1253/circj.CJ-13-1579

All rights are reserved to the Japanese Circulation Society. For permissions, please e-mail: cj@j-circ.or.jp 


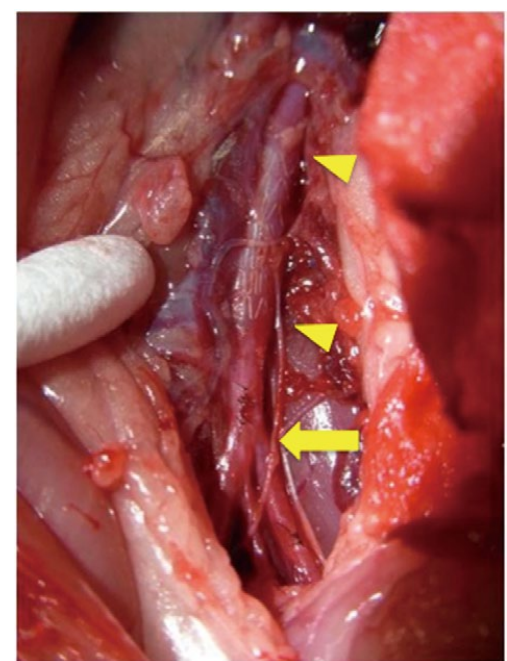

Figure 1. A representative photograph showing a stent-deployed rat abdominal aorta. The arrow indicates the aortic bifurcation, and the arrowheads indicate both ends of the deployed stent.

that adrenomedullin (AM) produced by ASC is involved in the ASC-induced restoration of erectile function. ${ }^{9,10}$ Because AM also exhibits potent proangiogenic activity, ${ }^{11-13}$ it is possible that ASC could stimulate reendothelialization in stented vessels by producing proangiogenic cytokines such as Ang-1 and AM.

Endothelial injury models such as balloon injury of the carotid artery and wire injury of the femoral artery have been widely used to analyze the mechanisms of restenosis of the coronary artery. In contrast to artery stenting, where the implanted stent induces inflammation persistently in the vessel wall, injury to endothelial cells in these models is transient. We, therefore, used the stenting model of the rat abdominal aorta in this study. There are several merits to use rats. First, the diameter of rat aorta is suitable to deploy coronary stents that are clinically used. Second, there are established rat disease models such as hypertension, obesity and diabetes. Therefore, it is possible to analyze neointimal formation and reendothelialization in disease models. In this study, we deployed BMS and DES in the rat abdominal aorta and examined whether ASC administration stimulated reendothelialization in these models.

\section{Methods}

\section{Reagents}

Anti-VE-cadherin antibody used for immunohistochemical analysis was obtained from LifeSpan BioSciences (Seattle, WA, USA).

\section{Cell Culture}

ASC were cultured from male Wistar rats as we have previously reported. ${ }^{7}$ ASC were first cultured in a 1:1 mixture of Dulbecco's modified Eagle medium and F12 medium containing 10\% fetal bovine serum (FBS), and split several times to expand the cells. Passages 2 to 4 were used for the experiments. ASC were then cultured on fibronectin-coated dishes in endothelial growth medium-2MV (EGM; Lonza Walkersville, Inc, Walkersville, MD, USA) for 1 week, because ASC cultured in this medium produce several proangiogenic cytokines such as Ang-1 and $\mathrm{AM}^{8,9}$ and effectively stimulate reendothelialization in a

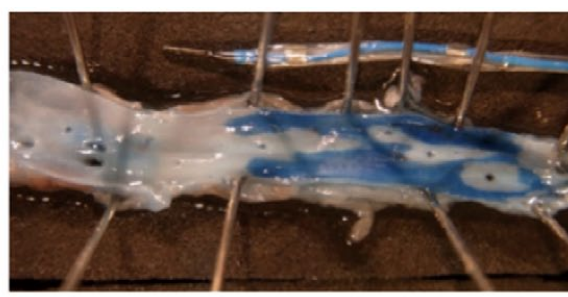

\section{Cont}

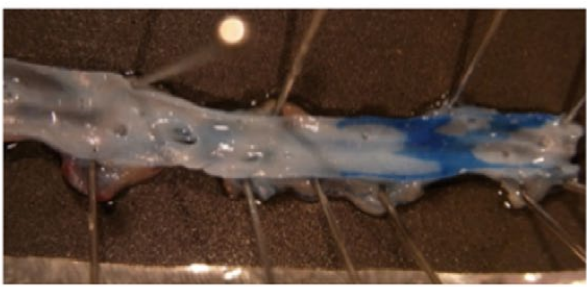

ASC

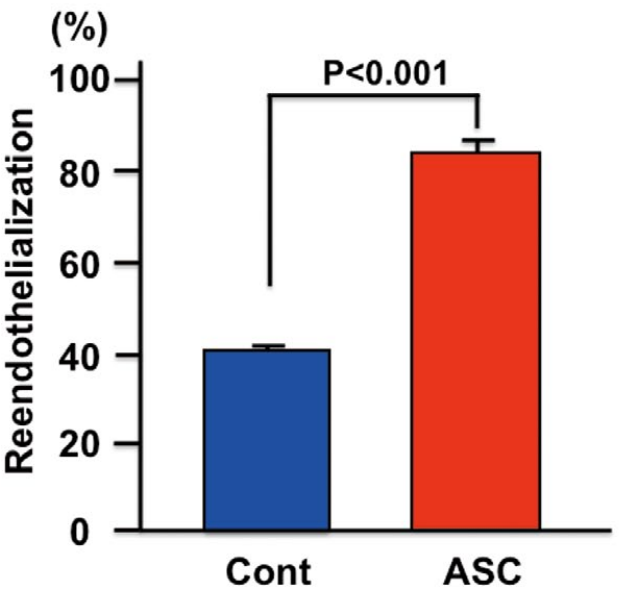

Figure 2. Adipose tissue-derived stem cells (ASC) stimulate reendothelialization in balloon-injured abdominal aorta. A balloon catheter was introduced into the abdominal aorta and inflated to induce endothelial injury. ASC were cultured in endothelial growth medium-2MV (EGM) on UpCell cell culture dishes for 1 week to prepare sheet-like lumps of ASC. An ASC sheet containing $1 \times 10^{6}$ cells was placed around the abdominal aorta, and the aorta was harvested 14 days after the balloon injury to assess reendothelialization by Evans blue staining. The reendothelialization rate was compared between control aorta (Cont; without ASC administration) and aorta covered with the ASC sheet (ASC) ( $n=8$ per group).

wire injury model of rat femoral artery, as we previously reported. ${ }^{7}$ EGM consists of endothelial basal medium-2 (Lonza Walkersville, Inc, Walkersville, MD, USA) containing 5\% FBS plus growth factors such as epidermal growth factor, hydrocortisone, vascular endothelial growth factor (VEGF)-A, basic fibroblast growth factor (bFGF) and insulin-like growth factor-1. To harvest ASC as one sheet and not a single-celled suspension, we used UpCell cell culture dishes (CellSeed, Tokyo, Japan). These dishes contain a temperature-sensitive extracellular matrix that is hydrophobic at temperatures more than $32^{\circ} \mathrm{C}$ and hydrophilic at temperatures less than $32^{\circ} \mathrm{C}$. Thus, ASC could be harvested as a sheet-like lump at room temperature without trypsinization. In some experiments, ASC were labeled with PKH26 (a red fluorescent dye; Sigma-Aldrich, St. Louis, MO, USA) according to the protocol provided by the manufacturer 

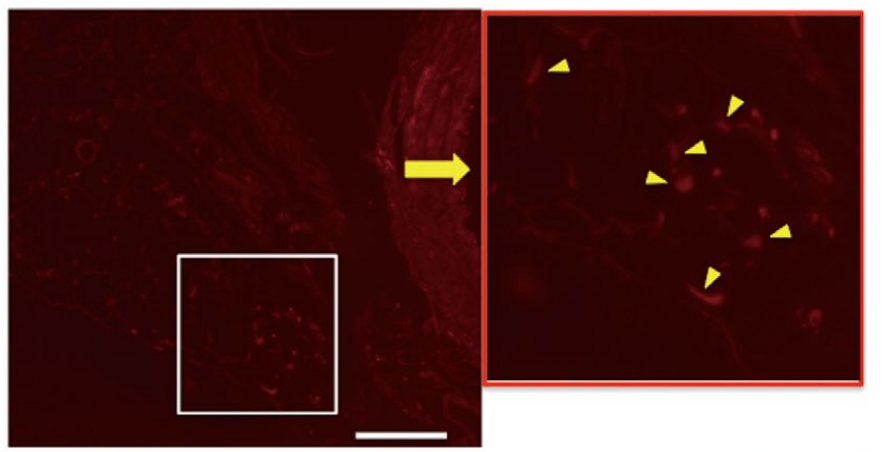

\section{Day 4}
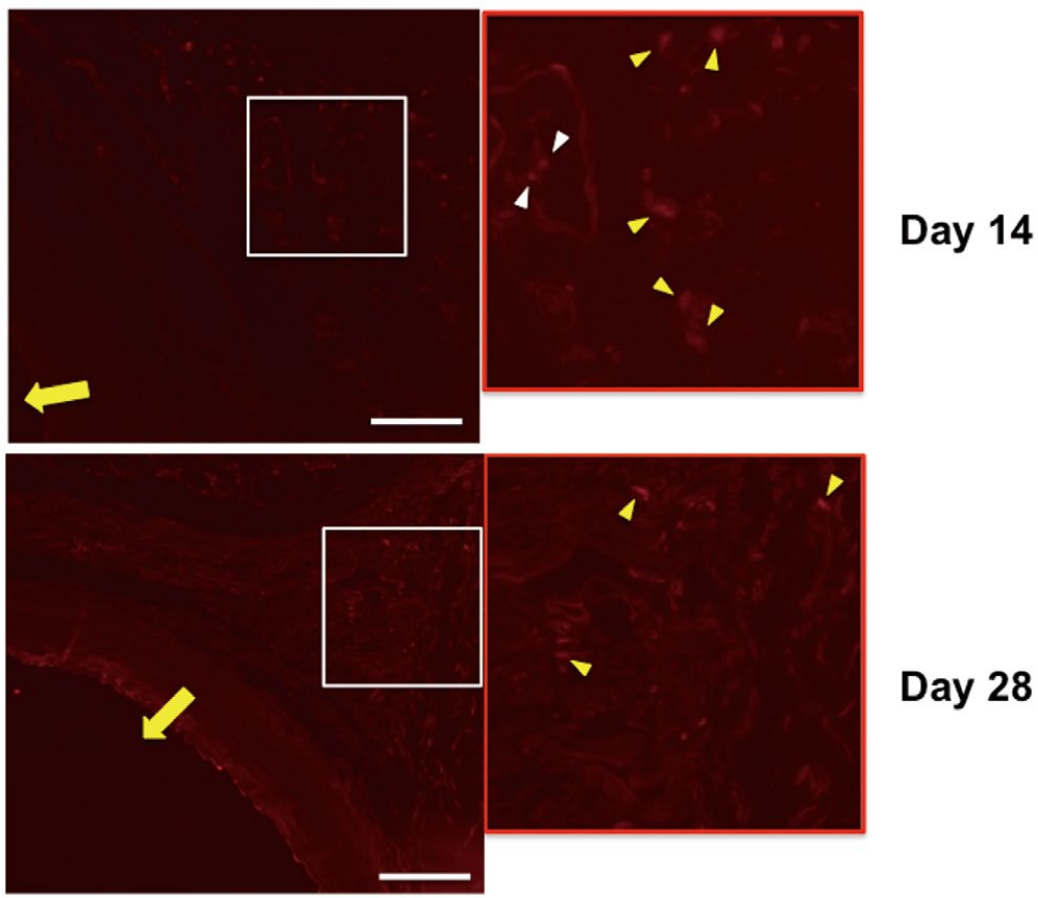

Figure 3. The fate of adipose tissue-derived stem cells (ASC) administered around the abdominal aorta. ASC were labeled with PKH26 red fluorescence dye and cultured in endothelial growth medium-2MV (EGM) on UpCell cell culture dishes for 1 week to prepare sheet-like lumps of ASC. ASC sheets were placed around the abdominal aorta and the aorta was harvested 4,14 and 28 days after ASC administration to examine the fate of ASC. The areas boxed with white squares are enlarged in the red squares on the right side. Arrows indicate the lumen of the abdominal aorta, and yellow arrowheads indicate $\mathrm{PKH} 26$ positive ASC. White arrowheads indicate the autofluorescence of red blood cells. Bars are $100 \mu \mathrm{m}$.

to monitor the cells, and they were then plated on UpCell cell culture dishes to harvest an ASC sheet. This sheet was placed around the rat abdominal aorta.

\section{Balloon Injury and Stent Implantation}

The institutional committee for animal research of the Tokyo University approved all procedures involving experimental animals. Stent deployment was performed as previously reported, with modifications. ${ }^{14}$ Male Wistar rats (8-10 weeks old) were anesthetized using an intraperitoneal injection of pentobarbital and a midline incision was made to approach the abdominal aorta. The abdominal aorta was isolated from the surrounding tissue, and a vascular clamp was placed onto the aorta distal to the renal arteries. A small incision was then made in the distal abdominal aorta proximal to the aortic bifurcation, and a balloon catheter was inserted and inflated to deploy a pre-mounted stent. The balloon was inflated such that the ratio of the stent diameter to the aorta diameter was 1.1. The balloon was usually inflated at a low pressure of 4-6 atm to achieve this ratio. After deflation and removal of the balloon, the aortic incision was closed with a 9-0 suture (Figure 1). Reperfusion was then established by removing the clip. The ASC sheet was placed around the aorta at this time point. We used $2.25 \times 8 \mathrm{~mm}$ Driver coronary stents (Medtronic Japan Co, Ltd, Tokyo, Japan) and 2.5×8 mm Cypher stents (Johnson \& Johnson Japan Inc, Tokyo, Japan). To induce balloon injury only, the balloon catheter, without stent mounting, was inserted and inflated to $6 \mathrm{~atm}$. The abdominal aorta was harvested 7, 14, and 28 days after stenting for histological analysis.

\section{Evans Blue Staining}

To evaluate reendothelialization, $0.5 \%$ Evans blue dye $(0.5 \mathrm{ml})$ was injected into the tail vein. After $10 \mathrm{~min}$, the abdominal aorta was perfusion-fixed, excised, opened by longitudinally cutting in half, and its image was photographed. The reendothelialization rate was calculated by quantifying the ratio of the white area (reendothelialized area) to the white area plus the blue area (endothelium-denuded area).

\section{Histochemistry}

The abdominal aorta was fixed by perfusion with $4 \%$ paraformaldehyde. The abdominal aorta with stenting was embedded in a Technovit 8100 (Okenshoji, Tokyo, Japan), a glycol methacrylate resin, according to the protocol provided by the manufacturer. Cross-sections ( $5 \mu \mathrm{m}$ ) were cut every $1 \mathrm{~mm}$ from the end region of the stent using a TC-65 tungsten blade (Leica 
A

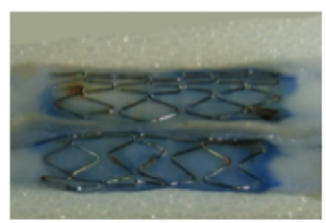

Cont Day 14

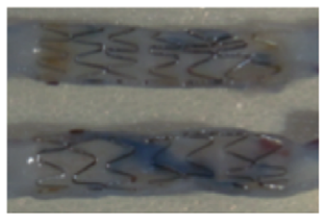

Cont Day 28

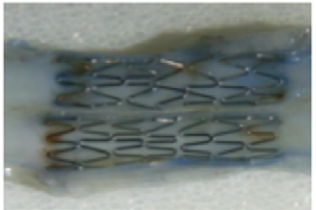

ASC Day 14

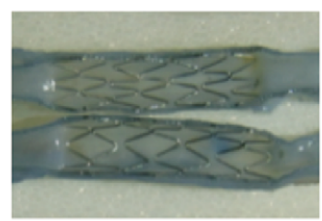

ASC Day 28
C

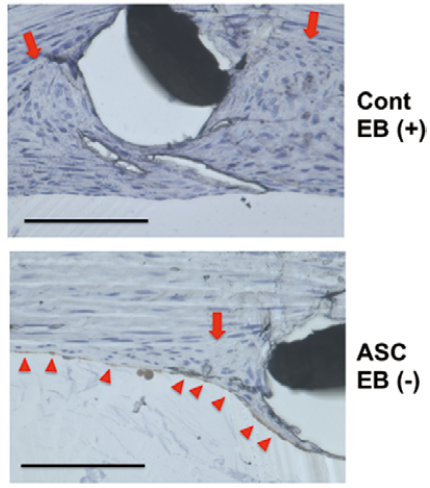

B

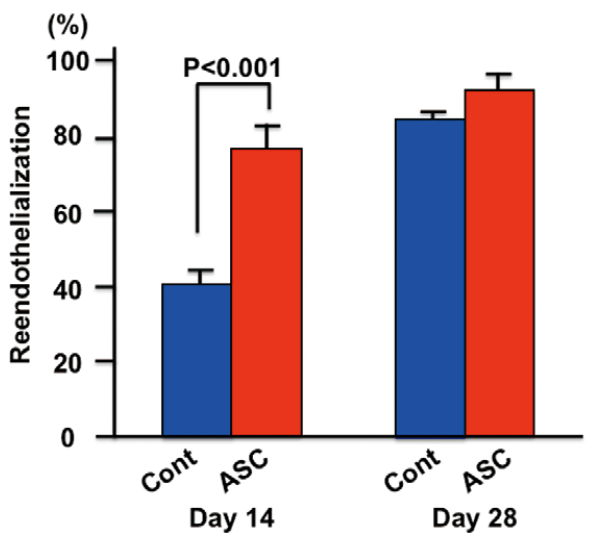

Figure 4. Adipose tissue-derived stem cells (ASC) stimulate reendothelialization in bare metal stents (BMS)-implanted abdominal aorta. A Driver stent was deployed in the abdominal aorta. ASC cultured in endothelial growth medium-2MV (EGM) on UpCell cell culture dishes for 1 week were collected as a sheet-like lump and placed around the abdominal aorta. The aorta was harvested 14 days and 28 days after stent deployment. Each aorta was cut in half longitudinally and the reendothelialization rate was compared between control aorta (Cont; without ASC administration) and aorta covered with ASC sheet (ASC). (A) Representative photographs of Evans blue staining of the abdominal aorta. (B) Statistical analysis of the reendothelialization rate ( $n=6$ per group). (C) To analyze the accuracy of Evans blue staining, the Evans blue positive area (EB (+); stained blue) from control aorta (Cont) and the Evans blue negative area (EB (-); remained white) from aorta covered with ASC sheets (ASC) were immunostained with anti-VE cadherin antibody, embedded in resin, sliced and photographed. Arrows indicate the border of the neointimal layer and medial layer. Arrowheads indicate the VE cadherin-positive endothelial layer. Bars are $100 \mu \mathrm{m}$.

Microsystems, Tokyo, Japan), and stained with hematoxylin and eosin. For immunohistochemistry, the whole abdominal aorta was incubated with a primary antibody reactive to VEcadherin in a microtube. The abdominal aorta was then incubated with a biotinylated secondary antibody and finally horseradish peroxidase-labeled streptavidin, following the instructions provided by the manufacturer (DAKO, Tokyo, Japan). The abdominal aorta was then embedded in the Technovit 8100 and processed as described above. The intima to medial ratio (I/M ratio) was calculated by measuring the areas of the neointimal and medial layers. The section of each aorta that showed maximal neointimal formation was used for statistical analysis; this section was located usually in the middle of the stent.

\section{Adenovirus}

The construction of adenovirus that expresses rat AM (AdAM) has been previously reported. ${ }^{9}$ A recombinant adenovirus that expresses GFP (AdGFP) was obtained from Quantum Biotechnologies (Montreal, Canada). Adenovirus at the amount of $1 \times 10^{9}$ plaque forming units was administered in the connective tissue around the abdominal aorta.

\section{Statistical Analysis}

The values are expressed as the mean \pm SEM. Statistical analyses were performed using analysis of variance followed by the Student-Neumann-Keuls test. Differences with a P value of $<0.05$ were considered to be statistically significant. 


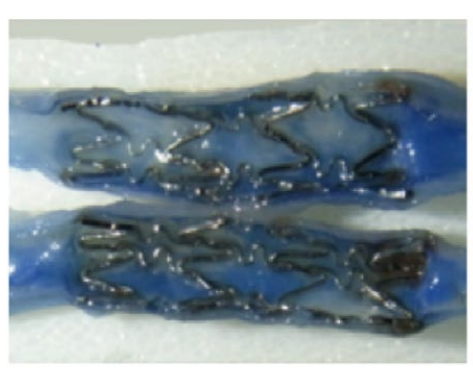

Cont Day 14

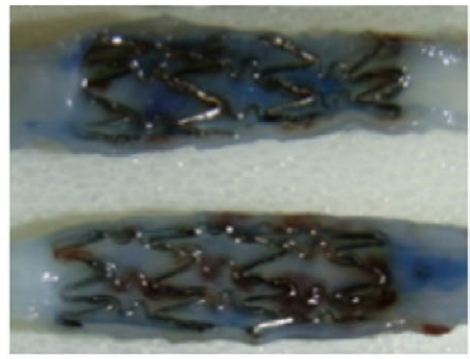

ASC Day 14

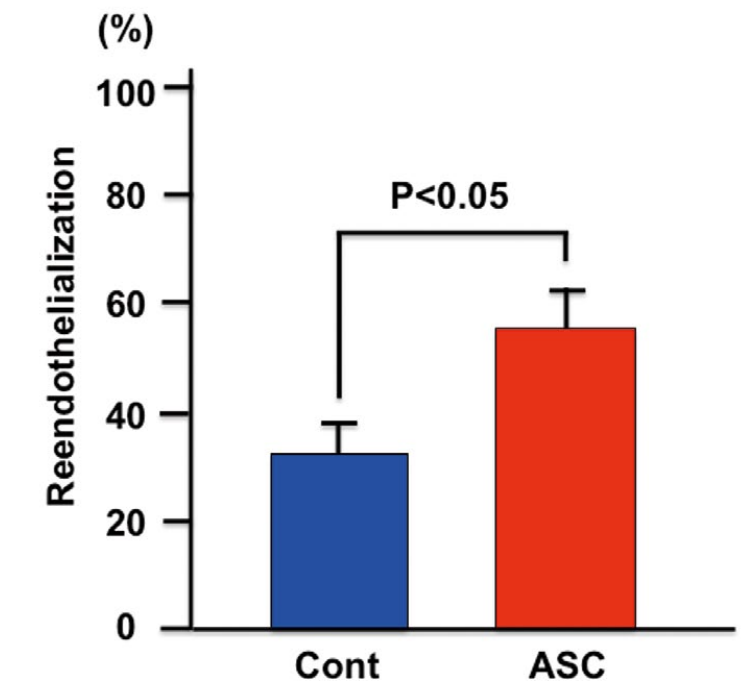

Figure 5. Adipose tissue-derived stem cells (ASC) stimulate reendothelialization in drugeluting stents (DES)-implanted abdominal aorta. Experiments were performed in a similar way as that described in Figure 4, except that Cypher stents were used instead of Driver stents. The aorta was harvested 14 days after stent deployment to analyze the reendothelialization rate ( $n=5$ per group).

\section{Results}

\section{ASC Stimulated Reendothelialization in Balloon-Injured Abdominal Aorta}

Because we have reported that ASC stimulate reendothelialization and inhibit neointimal formation in a wire injury model of the rat femoral artery when ASC are administered from the adventitial side, ${ }^{7}$ we administered ASC from the adventitial side of the abdominal aorta in this study. We administered ASC both as a single-celled suspension and as a sheet-like lump, and compared their effect on reendothelialization after balloon injury of the abdominal aorta. ASC administered in a sheet-like lump had a more potent effect on reendothelialization than the single-celled suspension (data not shown), probably because ASC remained around the aorta for a longer period of time. Therefore, we administered ASC in a sheet-like lump throughout this study. ASC significantly stimulated reendothelialization in balloon-injured abdominal aorta compared with balloon-injured aorta without the ASC sheet administration (Figure 2), suggesting that ASC potentially exert effects from the adventitial side in a paracrine manner in large vessels as well as in small vessels.

\section{Fate of ASC Sheets Placed Around the Abdominal Aorta}

We next assessed how long ASC remained around the aorta. Cells were labeled with PKH26 fluorescent dye and administered around the aorta in a sheet. ASC remained around the aorta until 14 days after administration (Figure 3). Although ASC were still detected 28 days after administration, their number remarkably decreased at this time point. However, ASC admin- istered in a sheet-like lump remained around the aorta for a longer time than those administered around the femoral artery in a single-celled suspension (3-7 days). ${ }^{8}$

\section{ASC Stimulated Reendothelialization in Stent-Implanted Abdominal Aorta}

To examine the effects of ASC on reendothelialization in stentimplanted abdominal aorta, we used 2 types of stents: a Driver coronary stent (bare metal), and a Cypher stent (sirolimus-eluting). ASC stimulated reendothelialization significantly 14 days after the Driver stent implantation compared with control aorta without ASC administration (Figures 4A,B). Because reendothelialization occurred naturally and efficiently in control aorta 28 days after stent implantation, no significant effect of ASC administration was observed at this time point. To confirm the accuracy of Evans blue staining, the Evans blue-positive region (blue) from the control aorta and Evans blue-negative region (white) from the ASC sheet-administered aorta were immunostained with anti-VE-cadherin antibody. However, we were unable to stain VECs in the aorta that was embedded in the Technovit 8100 resin (probably because the antibody did not penetrate the mesh of the resin sufficiently to reach VECs). In addition, we were unable to remove the resin after polymerization because it is insoluble in organic solvents such as xylene. Therefore, we pre-stained the whole aorta with anti-VEcadherin antibody and then embedded it in the resin for thin slicing. The Evans blue positive region was VE-cadherin negative, and the Evans blue negative region was VE-cadherin positive (Figure 4C). Thus, Evans blue staining appeared to indi- 


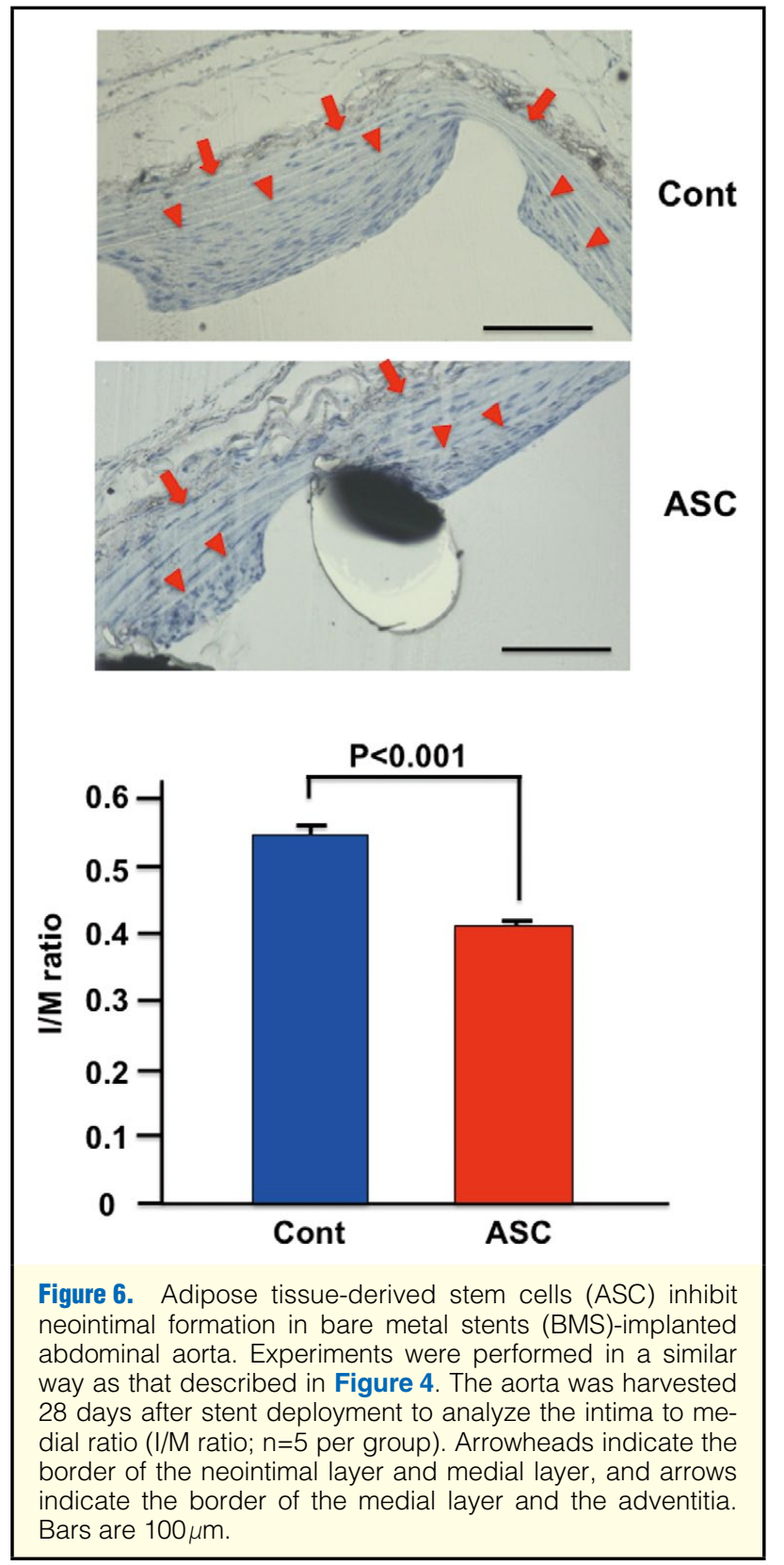

cate the presence and absence of VECs accurately. ASC also stimulated reendothelialization significantly at 14 days after Cypher stent implantation compared with the control aorta (Figure 5), although the effects were weaker than when ASC were administered around the Driver stent.

\section{ASC Inhibited Neointimal Formation in BMS-Implanted Abdominal Aorta}

We next examined whether ASC inhibited neointimal formation. Neointimal formation was observed in the Driver stent-implanted aorta, particularly around the stent struts at 28 days after stent implantation (Figure 6). ASC significantly suppressed neointimal formation compared with the control.

\section{AM Stimulated Reendothelialization and Inhibited Neointimal Formation in BMS-Implanted Abdominal Aorta} We have reported that ASC produce AM, particularly when

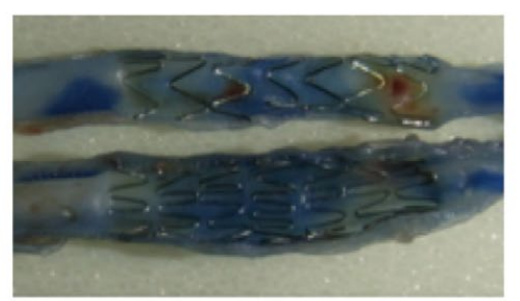

\section{AdGFP}

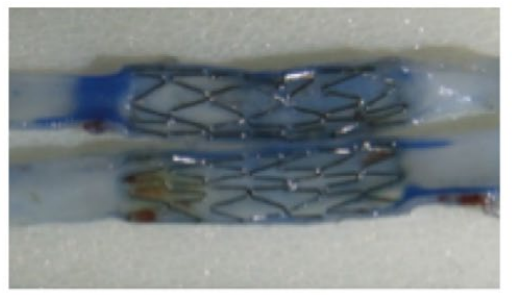

AdAM

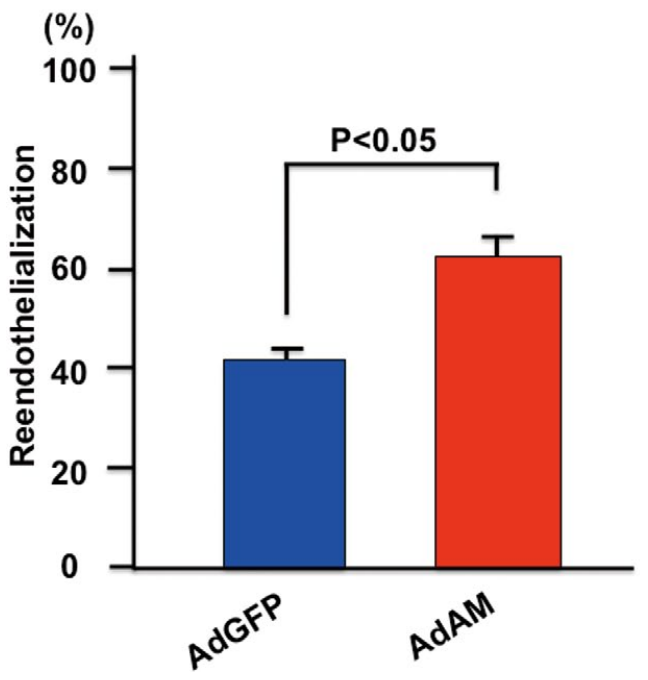

Figure 7. Adrenomedullin (AM) stimulates reendothelialization in bare metal stents (BMS)-implanted abdominal aorta. After deployment of a Driver stent in the abdominal aorta, suspensions of adenovirus that expresses green fluorescent protein (AdGFP) or adenovirus that expresses AM (AdAM) were injected into the connective tissue surrounding the abdominal aorta. The aorta was harvested 14 days after stent deployment to analyze the reendothelialization rate $(n=5$ per group).

cultured in EGM. ${ }^{9}$ Furthermore, it has been reported that AM has potent proangiogenic activities. ${ }^{11-13}$ Therefore, we infected AdAM from the adventitial side of the abdominal aorta and examined whether AM stimulated reendothelialization in Driver stent-implanted aorta. AdAM infection significantly stimulated reendothelialization compared with AdGFP infection (Figure 7). AdAM infection also significantly suppressed neointimal formation compared with AdGFP infection (Figure 8). We also used an adenovirus that expresses rat Ang-1 (AdAng-1), because we have reported that ASC produce Ang- $1 .^{8}$ AdAng- 1 infection did not significantly stimulate reendothelialization in Driver stent-implanted aorta (data not shown), although it significantly stimulated reendothelialization in a wire injury model of rat femoral artery. ${ }^{8}$ 


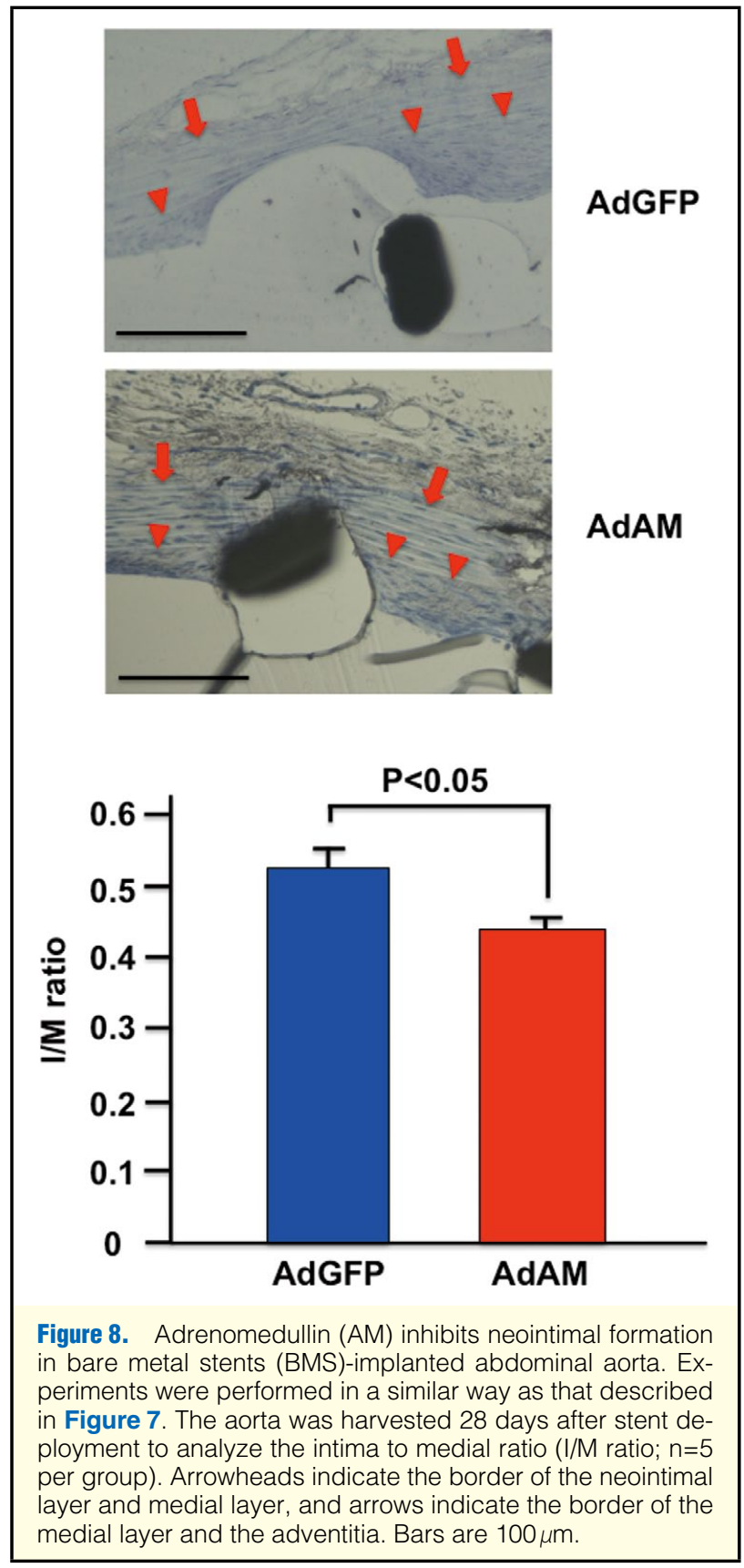

\section{Discussion}

In this study, we administered ASC from the adventitial side of the abdominal aorta, and found that ASC stimulated reendothelialization and inhibited neointimal formation in stent-implanted rat abdominal aorta. We have demonstrated that ASC administered from the adventitial side stimulate reendothelialization and inhibit neointimal formation in a wire injury model of rat femoral artery. ${ }^{8}$ We have also demonstrated that Ang-1 secreted from ASC is implicated in these effects of ASC. ${ }^{8}$ Therefore, ASC also seem to stimulate reendothelialization and inhibit neointimal formation in a paracrine manner in stent-implanted rat abdominal aorta.

Endothelial injury models such as the balloon injury model of the carotid artery and the wire injury model of the femoral artery have been widely used to analyze the mechanisms of restenosis of the coronary artery. The injury to the endothelium occurs transiently in these models. In contrast, stent implantation causes persistent inflammation to tissues around the stent, because a stent is a foreign material and it continually stretches the vascular wall. It is, therefore, necessary to use an animal model in which a coronary stent is actually deployed in a vessel. Although the rabbit iliac artery and the pig coronary artery are frequently used for stenting, ${ }^{15}$ we used the rat abdominal aorta in this study to analyze the effects of ASC. There are several merits to use rats. First, it is easier to treat rats than bigger animals. Second, there are several disease models in rats such as hypertension, obesity and diabetes. Therefore, it is possible to study the mechanisms of restenosis in these diseases. ASC stimulated reendothelialization effectively in BMS-implanted aorta. Although ASC significantly stimulated reendothelialization in DES-implanted aorta, their effect was weaker than that observed in BMS-implanted aorta, probably because sirolimus eluting from the stent exerted potent anti-migratory and anti-proliferative effects on VECs. Designing stents that elute proangiogenic factors rather than anti-proliferative drugs might be a promising strategy to stimulate reendothelialization and inhibit neointimal formation in the future.

Among the cytokines that ASC produce, AM stimulated reendothelialization and inhibited neointimal formation in BMSimplanted aorta. Consistent with this, it has been reported that AM stimulates angiogenesis and inhibits neointimal formation. ${ }^{11-13,16-18}$ Therefore, AM might be a cytokine that mediated ASC-induced stimulation of reendothelialization and inhibition of neointimal formation. Although Ang-1 stimulated reendothelialization and inhibited neointimal formation in the wire injury model of the rat femoral artery, ${ }^{8}$ it did not significantly stimulate reendothelialization in the BMS-implanted aorta (data not shown). The reason why AM had a more potent effect on the stimulation of reendothelialization than Ang-1 in stented aorta remains unclear. However, there are several possible explanations. First, It was reported that AM transactivated the type 2 receptor for VEGF. ${ }^{19}$ Therefore, AM might stimulate proliferation and migration of VECs via the type 2 receptor for VEGF as well as via the receptor for AM. Second, AM reportedly stimulated the production of VEGF and bFGF in the brain. ${ }^{20}$ Thus, VEGF and bFGF might stimulate proliferation and migration of VECs cooperatively with AM. Third, it was demonstrated in adrenocortical cells that VEGF and AM stimulated the expression of AM and VEGF, respectively. ${ }^{21}$ There might be a positive feedback loop in the production of AM and VEGF. Furthermore, it was demonstrated that AM inhibited the proinflammatory effects of VEGF such as the induction of the expression of intercellular adhesion molecule-1, vascular cell adhesion molecule-1 and E-selectin. ${ }^{22}$ Therefore, AM might cooperate with VEGF to stimulate proliferation and migration of VECs without inducing inflammation in the tissues. Our results support our hypothesis that an artery stenting model is required to analyze the mechanisms of coronary restenosis and explore strategies for the treatment of coronary restenosis. Treatment with a combination of several proangiogenic cytokines might be necessary in the future to stimulate reendothelialization and inhibit neointimal formation more potently.

It is well established that macrophages play pivotal roles in neointimal formation. ${ }^{23-25}$ Macrophages could not be immunostained in the present study, probably because antibodies did not penetrate the resin we used in this study. In addition, it was difficult to remove the resin because it was insoluble in organic solvents such as xylen. However, the infiltration of monocytes and lymphocytes around the stent struts, as assessed by hema- 
toxylin and eosin staining, was remarkably suppressed by ASC administration in BMS-implanted aorta (data not shown). Thus, another mechanism by which ASC inhibited neointimal formation might be by inhibiting the infiltration of inflammatory cells such as monocytes and macrophages. Previous reports demonstrated that both AM and Ang-1 have activities to inhibit macrophage infiltration and the production of proinflammatory cytokines from macrophages. ${ }^{26-29}$ Therefore, it is possible that AM and/or Ang-1 mediated the ASC-induced suppression of leukocyte infiltration. Nevertheless, future studies are required to assess this hypothesis.

In summary, ASC administered from the adventitial side significantly stimulated reendothelialization and inhibited neointimal formation in stent-deployed rat abdominal aorta. The design of stents that elute several proangiogenic and anti-inflammatory cytokines might be a promising strategy to inhibit restenosis and late thrombosis after stent deployment in the coronary artery.

\section{Acknowledgments}

We thank Medtronic Japan Co, Ltd and Johnson \& Johnson Japan Inc for supplying coronary stents at lower prices. We also thank Ms Asuka Ishii for her technical assistance.

\section{Disclosures}

This research was supported, in part, by a Grant-in-Aid for Specially Promoted Research (22000006) to T.N.

\section{References}

1. Bavry AA, Kumbhani DJ, Helton TJ, Borek PP, Mood GR, Bhatt DL. Late thrombosis of drug-eluting stents: A meta-analysis of randomized clinical trials. Am J Med 2006; 119: 1056-1061.

2. Stone GW, Moses JW, Ellis SG, Schofer J, Dawkins KD, Morice MC, et al. Safety and efficacy of sirolimus- and paclitaxel-eluting coronary stents. N Engl J Med 2007; 356: 998-1008.

3. Smith WL. Prostaglandin biosynthesis and its compartmentation in vascular smooth muscle and endothelial cells. Annu Rev Physiol 1986; 48: $251-262$.

4. Suga S, Nakao K, Itoh H, Komatsu Y, Ogawa Y, Hama N, et al. Endothelial production of c-type natriuretic peptide and its marked augmentation by transforming growth factor-beta: Possible existence of "vascular natriuretic peptide system". J Clin Invest 1992; 90: 1145 1149.

5. Nakanishi C, Nagaya N, Ohnishi S, Yamahara K, Takabatake S, Konno T, et al. Gene and protein expression analysis of mesenchymal stem cells derived from rat adipose tissue and bone marrow. Circ $J$ 2011; 75: 2260-2268.

6. Lee HC, An SG, Lee HW, Park JS, Cha KS, Hong TJ, et al. Safety and effect of adipose tissue-derived stem cell implantation in patients with critical limb ischemia: A pilot study. Circ J 2012; 76: 17501760.

7. Takahashi M, Suzuki E, Oba S, Nishimatsu H, Kimura K, Nagano $\mathrm{T}$, et al. Adipose tissue-derived stem cells inhibit neointimal formation in a paracrine fashion in rat femoral artery. Am J Physiol Heart Circ Physiol 2010; 298: H415-H423.

8. Takahashi M, Suzuki E, Kumano S, Oba S, Sato T, Nishimatsu H, et al. Angiopoietin-1 mediates adipose tissue-derived stem cell-induced inhibition of neointimal formation in rat femoral artery. Circ J 2013; 77: $1574-1584$.

9. Nishimatsu H, Suzuki E, Kumano S, Nomiya A, Liu M, Kume H, et al. Adrenomedullin mediates adipose tissue-derived stem cell-induced restoration of erectile function in diabetic rats. J Sex Med 2012; 9: $482-493$.

10. Nishimatsu H, Suzuki E, Nomiya A, Niimi A, Suzuki M, Fujimura $\mathrm{T}$, et al. Adrenomedullin and angiopoietin-1 additively restore erectile function in diabetic rats: Comparison with the combination therapy of vascular endothelial growth factor and angiopoietin-1. J Sex Med 2013; 10: 1707-1719.

11. Kim W, Moon SO, Sung MJ, Kim SH, Lee S, So JN, et al. Angiogenic role of adrenomedullin through activation of Akt, mitogen-activated protein kinase, and focal adhesion kinase in endothelial cells. FASEB
J 2003; 17: 1937-1939.

12. Tokunaga N, Nagaya N, Shirai M, Tanaka E, Ishibashi-Ueda H, Harada-Shiba M, et al. Adrenomedullin gene transfer induces therapeutic angiogenesis in a rabbit model of chronic hind limb ischemia: Benefits of a novel nonviral vector, gelatin. Circulation 2004; 109: $526-531$.

13. Iimuro S, Shindo T, Moriyama N, Amaki T, Niu P, Takeda N, et al. Angiogenic effects of adrenomedullin in ischemia and tumor growth. Circ Res 2004; 95: 415-423.

14. Langeveld B, Roks AJ, Tio RA, van Boven AJ, van der Want JJ, Henning RH, et al. Rat abdominal aorta stenting: A new and reliable small animal model for in-stent restenosis. J Vasc Res 2004; 41: $377-386$.

15. Johnson GJ, Griggs TR, Badimon L. The utility of animal models in the preclinical study of interventions to prevent human coronary artery restenosis: Analysis and recommendations: On behalf of the subcommittee on animal, cellular and molecular models of thrombosis and haemostasis of the scientific and standardization committee of the international society on thrombosis and haemostasis. Thromb Haemost 1999; 81: 835-843.

16. Imai Y, Shindo T, Maemura K, Sata M, Saito Y, Kurihara Y, et al. Resistance to neointimal hyperplasia and fatty streak formation in mice with adrenomedullin overexpression. Arterioscler Thromb Vasc Biol 2002; 22: 1310-1315.

17. Tsuruda T, Kato J, Matsui E, Hatakeyama K, Masuyama H, Imamura $\mathrm{T}$, et al. Adrenomedullin alleviates not only neointimal formation but also perivascular hyperplasia following arterial injury in rats. Eur $J$ Pharmacol 2005; 508: 201-204.

18. Rauma-Pinola T, Paakko P, Ilves M, Serpi R, Romppanen H, Vuolteenaho $\mathrm{O}$, et al. Adrenomedullin gene transfer induces neointimal apoptosis and inhibits neointimal hyperplasia in injured rat artery. J Gene Med 2006; 8: 452-458.

19. Guidolin D, Albertin G, Spinazzi R, Sorato E, Mascarin A, Cavallo $\mathrm{D}$, et al. Adrenomedullin stimulates angiogenic response in cultured human vascular endothelial cells: Involvement of the vascular endothelial growth factor receptor 2. Peptides 2008; 29: 2013-2023.

20. Maki T, Ihara M, Fujita Y, Nambu T, Harada H, Ito H, et al. Angiogenic roles of adrenomedullin through vascular endothelial growth factor induction. Neuroreport 2011; 22: 442-447.

21. Albertin G, Rucinski M, Carraro G, Forneris M, Andreis P, Malendowicz LK, et al. Adrenomedullin and vascular endothelium growth factor genes are overexpressed in the regenerating rat adrenal cortex, and AM and VEGF reciprocally enhance their mRNA expression in cultured rat adrenocortical cells. Int J Mol Med 2005; 16: 431435.

22. Kim W, Moon SO, Lee S, Sung MJ, Kim SH, Park SK. Adrenomedullin reduces VEGF-induced endothelial adhesion molecules and adhesiveness through a phosphatidylinositol 3'-kinase pathway. Arterioscler Thromb Vasc Biol 2003; 23: 1377-1383.

23. Furukawa Y, Matsumori A, Ohashi N, Shioi T, Ono K, Harada A, et al. Anti-monocyte chemoattractant protein-1/monocyte chemotactic and activating factor antibody inhibits neointimal hyperplasia in injured rat carotid arteries. Circ Res 1999; 84: 306-314.

24. Danenberg HD, Fishbein I, Gao J, Monkkonen J, Reich R, Gati I, et al. Macrophage depletion by clodronate-containing liposomes reduces neointimal formation after balloon injury in rats and rabbits. Circulation 2002; 106: 599-605.

25. Mori E, Komori K, Yamaoka T, Tanii M, Kataoka C, Takeshita A, et al. Essential role of monocyte chemoattractant protein-1 in development of restenotic changes (neointimal hyperplasia and constrictive remodeling) after balloon angioplasty in hypercholesterolemic rabbits. Circulation 2002; 105: 2905-2910.

26. Kim I, Moon SO, Park SK, Chae SW, Koh GY. Angiopoietin-1 reduces VEGF-stimulated leukocyte adhesion to endothelial cells by reducing ICAM-1, VCAM-1, and e-selectin expression. Circ Res 2001; 89: $477-479$.

27. Gonzalez-Rey E, Chorny A, Varela N, Robledo G, Delgado M. Urocortin and adrenomedullin prevent lethal endotoxemia by downregulating the inflammatory response. Am J Pathol 2006; 168: 1921 1930.

28. Yanagawa B, Kataoka M, Ohnishi S, Kodama M, Tanaka K, Miyahara $\mathrm{Y}$, et al. Infusion of adrenomedullin improves acute myocarditis via attenuation of myocardial inflammation and edema. Cardiovasc Res 2007; 76: 110-118.

29. Gu H, Cui M, Bai Y, Chen F, Ma K, Zhou C, et al. Angiopoietin-1/ Tie2 signaling pathway inhibits lipopolysaccharide-induced activation of RAW264.7 macrophage cells. Biochem Biophys Res Commun 2010; 392: $178-182$. 